\title{
Editorial
}

\section{Umgang mit Elektroaltgeräten}

In Österreich wird seit 25 Jahren die Diskussion geführt, wie Elektroaltgeräte am besten gesammelt und verwertet werden können. Neben diesen Kernthemen sind auch eine Produktgestaltung nach ökologischen Kriterien - Ecodesign sowie die Wiederverwendung und Reparatur wichtige Komponenten des Umgangs mit diesem Produktstrom. Seit mehr als 15 Jahren gibt es in Österreich Regelungen für Elektroaltgeräte, im Wesentlichen die EAG-Verordnung, ergänzend die RoHS-Bestimmungen und weitere Regelwerke. Wenn man hier ein Resümee ziehen möchte, ist festzuhalten, dass in Österreich funktionierende Sammel- und Verwertungssysteme eingerichtet wurden, die getrennte Sammlung im Wesentlichen durch die Kommunen - etabliert wurde und die Behandlung überwiegend im Inland stattfindet. Kritisch anzumerken ist, dass sowohl in der Sammlung als auch in der Verwertung von EAG ein systemischer Anreiz zum Ausbau der Sammlung als auch zur innovativen Erweiterung der Behandlung, beispielsweise zur Rückgewinnung von kritischen Rohstoffen, fehlt. Wiederverwendung und Reparatur spielen weiterhin eine untergeordnete Rolle und auch bezüglich Ecodesign sind nur kleine Fortschritte zu beobachten.

In dieser Ausgabe der Österreichischen Wasser- und Abfal/wirtschaft finden Sie diesbezüglich Beiträge zur Produktgestaltung, zum Thema Schadstoffe in Elektroaltgeräten, zu Re-Use und Reparatur von Elektrogeräten und zum Verwertungsprozess von Elektrokleingeräten. Die internationale Dimension wird anhand der Entwicklungen in Lateinamerika vorgestellt.

Roitner et al. stellen in Zusammenhang mit der Produktgestaltung einen Bewertungsansatz mittels statistischer Entropie vor, mit dem die Recyclingfähigkeit von Produkten bestimmt werden kann. Dies wird am Beispiel von Mobiltelefonen dargestellt.

Hawlik et al. gehen auf das Thema der Schadstoffe anhand der bromierten Flammhemmer ein und zeigen, dass Tastaturen, die mit mittlerweile nicht mehr zulässigen BFRs ausgestattet sind, in der Nutzung einen geringen, aber signifikanten Austrag durch simulierten Hautkontakt zeigen. Die Ergebnisse aus den Versuchen unter Deponiebedingungen legen nahe, dass es bei der Ablagerung derartiger Kunststoffe ohne Vorbehandlung nur zu einer geringen Auslaugung der bromierten Flammhemmer kommt.

Wiederverwendung (Re-Use) und Reparatur als wichtige Strategie zur Verlängerung der Produktnutzungsdauer und damit zur Verringerung der Abfallmengen werden im Beitrag von Wagner et al. analysiert. Dazu werden das Value Hill-Modell und die bestehenden Initiativen und Einrichtungen in Österreich vorgestellt.

Auf die Optimierung von Prozessen zur Verwertung von Elektrokleingeräten wird im Beitrag von Beigl und Salhofer eingegangen. Mit einer genaueren Bestimmung der Materialzusammensetzung von Elektrokleingeräten können die mechanischen Aufbereitungsschritte gezielter angewandt werden.

Die internationale Perspektive dieses globalen Themas wird am Beispiel Lateinamerikas gezeigt. Anders als in Europa gibt es in Lateinamerika kaum Regelungen und die Sammlung und Verwertung erfolgt zu einem großen Teil durch den informellen Sektor. Anhand von Nicaragua und Brasilien wird die bisherige Entwicklung und der Ausbildungsbedarf erläutert. 


\section{Editorial}

ao. Univ.-Prof. DI Dr. S. Salhofer ( $₫)$

Department Wasser-Atmosphäre-Umwelt,

Institut für Abfallwirtschaft, Universität

für Bodenkultur Wien,

Muthgasse 107,

1190 Wien, Österreich

stefan.salhofer@boku.ac.at 